\title{
The X-ray absorber of PKS 2126-158
}

\author{
F. Fiore ${ }^{1}$, M. Elvis ${ }^{2}$, R. Maiolino ${ }^{3}$, F. Nicastro ${ }^{2}$, A. Siemiginowska ${ }^{2}$, G. Stratta ${ }^{1,4}$, and V. D’Elia ${ }^{1}$ \\ 1 INAF - Osservatorio Astronomico di Roma, via Frascati 33, Monteporzio-Catone (RM), 00040, Italy \\ e-mail: stratta@mporzio.astro.it; delia@mporzio.astro.it \\ 2 Harvard-Smithsonian Center for Astrophysics, Cambridge MA 02138, USA \\ e-mail: elvis@head-cfa.harvard.edu; aneta@head-cfa.harvard.edu \\ 3 INAF - Osservatorio Astrofisico di Arcetri, largo E. Fermi, 5. 50125, Firenze, Italy \\ e-mail: maiolino@arcetri.astro.it \\ ${ }^{4}$ Universitá di Roma "La Sapienza", Piazzale Aldo Moro 19050 5, 00185 Roma, Italy
}

Received 8 January 2003 / Accepted 16 June 2003

\begin{abstract}
BeppoSAX observed the $z=3.27$ quasar PKS 2126-158 on May 24-28 1999 when its $2-10 \mathrm{keV}$ and $0.1-2.5 \mathrm{keV}$ fluxes were $1.1 \times 10^{-11}$ and $4.4 \times 10^{-12} \mathrm{erg} \mathrm{cm}^{-2} \mathrm{~s}^{-1}$ respectively, a factor of 2 higher than in all previous ROSAT and ASCA observations and $40 \%$ higher than in two more recent Chandra and XMM-Newton observations. The shortest detected rest frame variability timescale is of a few months, comparable to the causal timescale associated with an emission region of $\sim 10$ Schwarzschild radii around a few $\times 10^{10} M_{\odot}$ black hole. The source is detected with a signal to noise ratio $S / N \gtrsim 3$ up to $\sim 50 \mathrm{keV}, 215 \mathrm{keV}$ rest frame. The BeppoSAX observations confirm the presence of low energy absorption along the line of sight, independent of the continuum model adopted, at a high confidence level. Despite the limited spectral resolution of the BeppoSAX LECS and MECS it is possible to put constraints on different absorption and continuum models, but not to unambiguously determine the redshift of the absorber. If the absorber is not significantly ionized the BeppoSAX data do prefer an absorber at $z \lesssim 2.7$. Strong and complex metal line systems along the line of sight to PKS 2126-158 have been found at $z=0.6631$ and at $2.64<z<2.82$. They could well be associated with the X-ray absorption. Conversely, an ionized ("warm") absorber at the quasar redshift provides a good fit only if the iron abundance is smaller than $\sim 0.3$ solar, while that of the other elements is fixed to the solar value. Lower iron metallicity would imply a lower dust to gas ratio, since iron aggregates easily in dust. This can help in solving the apparent paradox of the lack of significant ultraviolet reddening in this source while strong absorption is detected in X-rays. Low iron abundance would be at odds with the supersolar abundances derived from the broad emission lines.
\end{abstract}

Key words. quasars: general - X-rays: galaxies

\section{Introduction}

The high redshift $(z>3)$ Universe can be probed by observing distant convenient "lighthouses" such as quasars, Gamma Ray Bursts, and luminous galaxies. Both emission and absorption features superimposed onto the source continuum can be used for this purpose. High quality spectra of high redshift cosmic sources can tell us about the physics and chemistry of the matter along the line of sight (i.e. density, physical state and element abundances). In particular, high- $z$ quasars and galaxies give a unique chance of probing the metallicity of the gas enriched by the first generations of stars. Iron is expected to be produced mostly by type Ia $\mathrm{SNe}$, occurring with a delay of $\approx 1 \mathrm{Gyr}$ with respect to the first type II SNe, which produce most of the $\alpha$ elements such as $\mathrm{O}, \mathrm{Si}, \mathrm{Mg}$. Therefore, the relative abundances of various elements produced on different

Send offprint requests to: F. Fiore,

e-mail: fiore@mporzio. astro.it timescale in high- $z$ quasars (e.g. the $[\alpha / \mathrm{Fe}]$ logarithmic ratio, where the columns are normalized to their solar value) is a sensitive clock of star-formation history, and its determination provides unique constraints on the star-formation and on the initial mass function at high redshift (e.g. Hamann \& Ferland 1999).

So far metallicities at high- $z$ have been studied by means of a few quasar and radio-galaxy UV rest-frame emission lines (see e.g. Hamann \& Ferland 1999; Constantin et al. 2002; Hamann et al. 2002), and via optical-UV absorption systems, both associated with the quasar and with galaxies which happen to lie along the line of sight to bright quasars.

Emission line metallicity determinations are plagued by uncertainties on the properties of the emitting gas (temperature, density, ion fraction, dust depletion) and by the small number of lines available to constrain the metallicity. In particular, the abundance of iron relative to the $\alpha$ elements is mostly inferred from the intensity of the UV Fe bump relative to MgII (2803 ̊) (Thompson et al. 1999; Iwamuro et al. 2002; 
Dietrich et al. 2002). However, the FeII UV bump is one of the most important coolants of the transition region of the BLR in AGNs and therefore might be characterized by a self-regulating intensity; indeed, Verner et al. (1999) have shown that the emissivity of the Fe UV bump is far from having a 1:1 relation with the iron abundance. As a consequence, the iron metallicity of high-z QSOs inferred by a naive comparison of the FeII-UV/MgII intensity at high and low redshift might be quite inaccurate or even deceiving.

Quasar intrinsic broad absorption line (BAL) metallicity determinations provide bizarre but usually supersolar metal abundances (Hamann 1998; Hamann \& Ferland 1999; Arav et al. 2001; Hamann et al. 2003). However, these could have been overestimated, because strong absorption lines like CIV could be optically thick and strongly saturated (e.g. Hamann et al. 2003). The analysis of BALs is complicated by the fact that usually the line troughs do not reach zero intensity, because the absorbers are leaky and/or because of scattered/emitted flux from the absorbing region. Furtheremore, from the opti$\mathrm{cal} / \mathrm{UV}$ BAL it is not possible to measure the abundances of iron, which is one of the key elements to constrain the star formation history.

In the case of absorption features not associated with the quasars, optical-UV metal line systems in galaxies along the line of sight to quasars probe mainly the ISM of outer haloes, rather than the bulge or the disk (see e.g. Churchill et al. 2000). Even the highest column density systems known, the Damped Ly $\alpha$ systems (DLAs), may give a distorted view of the bulk of the "typical galaxy" population at $z=2-4$. DLA studies are likely to be biased against dusty systems, since these would hide the background quasars in color-based surveys (e.g. Savaglio et al. 2000). Furthermore, in DLAs metallicities are usually evaluated using faint zinc lines, since iron, which is 20 times more abundant than zinc, is heavily depleted onto dust grains (see e.g. Pettini et al. 1997). Using zinc lines Pettini et al. (1999) and Prochaska \& Wolfe (1999) estimate a metallicity of about 0.1 at $z=3-4$. DLA metallicities may approach solar values at low- $z$ (Savaglio et al. 2000).

Summarizing, metallicity measurements at high redshift based on optical observations have often provided only partial or uncertain information. This statement applies specifically to iron. In contrast, X-ray measurements offer a unique means of investigating absorption systems because the X-ray absorption edges can constrain tightly the ionization state, the molecular state and the dust content of intervening material and so probe total column densities. A direct measurement of the depth of iron X-ray absorption edges will remove the degeneracy involving various physical parameters of the emitting region, which plague current metallicity estimates. This can be achieved at $z=3-4$ with the present instrumentation, because $\mathrm{Fe}-\mathrm{K}$ features at these redshift are redshifted to $1.5-2 \mathrm{keV}$, where the X-ray instrument sensitivity is the greatest.

This approach is plausible as there are strong signs of absorption toward several $z=2-3$ radio-loud quasars with implied column densities of $N_{\mathrm{H}} \sim 10^{22} \mathrm{~cm}^{-2}$ in the quasar rest frame (Elvis et al. 1994; Serlemitsos et al. 1994; Cappi et al. 1997; Fiore et al. 1998; Reeves \& Turner 2000). The quality of the ROSAT and ASCA discovery spectra however can not discriminate between a location for the absorber at the quasar or along the intervening line of sight. Fiore et al. (1998) and Elvis et al. (1998) argue for intrinsic origin because of the lack of absorbers in radio-quiet quasars at $z=2-3$. Kuhn et al. (2001) show that $z=3$ radio-quiet quasars have spectra that are as UV-bright as low redshift radio-quiet quasars, implying $A_{V} \lesssim 0.3$ mags. However, the absence of dust and of detectable X-ray $N_{\mathrm{H}}$ in $z=2-3$ radio quiet quasars could be a selection effect. Most quasars are selected via optical colors and a fairly modest reddening can drop the objects out of the samples. Furthermore, radio-quiet quasars are fainter in X-rays than radio-loud quasars and so have more poorly constrained spectra. So the issue of the location of the absorbers in radioloud quasars is still open.

PKS 2126-158 is the brightest quasar in X-rays at $z>3$, and it is the second brightest, after PKS 2149-306, at $z>2$. For this reason PKS 2126-158 has been studied in great detail at X-ray frequencies since the first Einstein detection (Worrall \& Wilkes 1990). Elvis et al. (1994) discovered a strong low energy cut-off, which is naturally explained by photoelectric absorption along the line of sight, in addition to the Galactic $21 \mathrm{~cm}$ column density of $4.85 \times 10^{20} \mathrm{~cm}^{-2}$ (Elvis et al. 1989). The implied column density ranges from $0.9-20 \times 10^{21} \mathrm{~cm}^{-2}$, if the absorber is at $z \sim 0$, it is not highly ionized and has solar abundances, up to a column of $0.8-2.7 \times 10^{22} \mathrm{~cm}^{-2}$ if the absorber is at $z \sim 3.27$, the quasar redshift. ASCA observations confirmed the low energy cutoff (Serlemitsos et al. 1994; Cappi et al. 1997; Reeves \& Turner 2000) but cannot distinguish beween genuine photoelectric absorption or continuum curvature. We have taken advantage of the good BeppoSAX sensitivity over a very broad band, from $0.1 \mathrm{keV}$ up to $200 \mathrm{keV}$, to disentangle photoelectric absorption and continuum curvature and to constrain the redshift, ionization state and metallicity of the absorber in PKS 2126-158.

The paper is organized as follows: Sect. 2 presents the observations and data reduction; Sect. 3 presents the spectral analysis; Sect. 4 discusses the main results on the low energy absorption.

\section{BeppoSAX data reduction and analysis}

BeppoSAX observed PKS 2126-158 from May 241999 through May 28 1999. The observations were performed with the BeppoSAX Narrow Field Instruments, LECS (0.1-10 keV, Parmar et al. 1997), MECS (1.3-10 keV, Boella et al. 1997b), HPGSPC (4-60 keV, Manzo et al. 1997) and PDS (13-200 keV, Frontera et al. 1997). LECS and MECS are imaging gas scintillation proportional counters, the HPGSPC is a collimated high pressure gas scintillation proportional counter and the PDS consists of four phoswich units. The PDS is operated in the so called "rocking mode", with a pair of units pointing to the source while the other pair monitor the background \pm 210 arcmin away. The units on and off source are interchanged every $96 \mathrm{~s}$. We report here the analysis of the LECS, MECS and PDS data; the HPGSPC data are very noisy, due to the high HPGSPC background. The MECS observations were performed with units 2 and 3 (on 1997 May 6th a technical failure caused unit MECS1 to be switched off); these data were 
Table 1. Observation log.

\begin{tabular}{lcc}
\hline \hline Instrument & Exposure (ks) & Count rate $^{a}$ \\
\hline LECS & 78.1 & $0.1-4 \mathrm{keV} \mathrm{5.50 \pm 0.09}$ \\
MECS & 107.9 & $1.7-10 \mathrm{keV} 11.9 \pm 0.10$ \\
PDS & 51.6 & $13-100 \mathrm{keV} 15.7 \pm 0.21$ \\
\hline
\end{tabular}

${ }^{a} 10^{-2}$ counts s ${ }^{-1}$.

combined after gain equalization. The LECS was operated during dark time only, therefore LECS exposure times are usually smaller than MECS ones. Table 1 gives the LECS, MECS and PDS exposure times and the count rates.

Standard data reduction was performed using the SAXDAS software package version 2.0 following Fiore et al. (1999). In particular, data are linearized and cleaned of Earth occultation periods (we accumulated data for Earth elevation angles $>5$ degrees) and unwanted periods of high particle background (satellite passages through the South Atlantic Anomaly and periods with magnetic cut-off rigidity $>6 \mathrm{GeV} / \mathrm{c}$ ). The low and stable particle background of the LECS, MECS and PDS (with variations at most of $30 \%$ around the orbit, due to the low inclination of the satellite orbit, 3.95 degrees), ensures that systematic errors in the subtraction of the background are negligible, i.e. smaller, in the energy bands of interest, than the statistical errors.

LECS and MECS spectra were extracted from regions of 8 arcmin and 3 arcmin radii respectively. These radii maximize the signal-to-noise ratio below $1 \mathrm{keV}$ in the LECS and above $2 \mathrm{keV}$ in the MECS. Background spectra were extracted in detector coordinates from high Galactic latitude "blank" fields (98_11 issue) using regions equal in size to the source extraction region. We have compared the mean level of the background in the LECS and MECS "blank fields" observations to the mean level of the background in the PKS 2126-158 observations using source free regions at various positions in the detectors. The "local" MECS background count rate is within $2 \%$ that in the "blank fields". On the other hand, for the LECS the "local" background count rate is $10 \%$ smaller than that of the "blank fields". For this reason, the "blank fields" background spectrum was multiplied by a factor of 0.9 before subtraction from the source spectrum.

The PDS data were reduced using the "variable risetime threshold" technique to reject particle background (see Fiore et al. 1999). This technique reduces the total $13-200 \mathrm{keV}$ background to about 20 counts $\mathrm{s}^{-1}$ and the $13-80 \mathrm{keV}$ background to about 6 counts s$^{-1}$ (instead of 30 and 10 counts s ${ }^{-1}$ respectively, obtained using the standard "fixed risetime threshold" technique).

The PDS rocking mode provides a reliable background subtraction. This can be checked by looking at the spectrum between 200 and $300 \mathrm{keV}$, where the effective area of the PDS to X-ray photons is small and therefore the source contribution is negligible. After background subtraction we obtain a count rate of $-0.0014 \pm 0.0100$ counts $\mathrm{s}^{-1}$, fully consistent with the expected value of 0 . The net (background subtracted) $13-100 \mathrm{keV}$ on-source signal is $0.157 \pm 0.021$ counts $\mathrm{s}^{-1}$.
The source is detected with a signal to noise ratio $S / N \gtrsim 3$ up to $\sim 50 \mathrm{keV}$, or $215 \mathrm{keV}$ in the rest frame). Confusion in the PDS collimator Field Of View (1.4 degrees FWHM) ultimately limits our capability to constrain the high energy spectrum. We have carefully checked for any possible contaminant in a region of 1.5 degrees radius around the source (using the NED, SIMBAD, AGN, clusters, CVs, Radio and X-ray sources catalogs) finding no obvious bright hard X-ray source. Of course there is the possibility of a bright "unknown" source in the PDS field of view. The chance of finding a source in any given 2 square degrees, the PDS beam area, is however small. The HEAO-1 A4 all sky catalog (Levine et al. 1984) lists just 7 high Galactic latitude sources in the $13-80 \mathrm{keV}$ band down to a flux of $2 \times 10^{-10} \mathrm{erg} \mathrm{cm}^{-2} \mathrm{~s}^{-1}$ (10 mCrab). The $13-80 \mathrm{keV}$ flux is about 20 times smaller than this figure and so, assuming a $\log N-\log S$ slope of -1.5 , we expect a chance coincidence rate of $2 \%$.

Spectral fits were performed using the XSPEC 11.0.1 software package and public response matrices as from the 1999 December release. For the MECS and PDS we used the standard on axis matrices (effective area and response matrix). For the LECS we built the effective area file corresponding to the source position in raw coordinates, since it turned out that this position is relatively far from the default pointing position (by about 3 raw pixels, i.e. 45 arcsec.) This caused the source to be closer than usual to one of the tungsten wires supporting the LECS window. Our LECS effective area file takes than correctly into account the photons absorbed by the wire.

LECS and MECS spectra were rebinned following two criteria: a) to sample the energy resolution of the detectors with four channels at all energies whenever possible, and b) to obtain at least 20 counts per energy channel.

Constant factors have been introduced in the fitting models in order to take into account the intercalibration systematics between the instruments (Fiore et al. 1999). Assuming the MECS as reference instrument, the expected factor between LECS and MECS is about 0.9 [0.7-1.1]. Fitting the LECS and MECS spectra in the common 1.7-4 keV band with a simple model produced a factor of $0.835 \pm 0.025$. In all following fits the LECS-MECS factor is therefore constrained to vary between 0.81 and 0.86 .

The expected factor between the PDS and MECS is 0.8 . We constrained the PDS normalization factor to vary in the range $0.7-0.9$, to allow for confusion in the PDS on-source and off-source collimators due to faint hard sources. The energy range used for the fits are: $0.1-4 \mathrm{keV}$ for the LECS (channels 12-400), 1.65-10 keV for the MECS (channels 37-220) and 13-200 keV for the PDS. Errors quoted in this paper are $90 \%$ confidence intervals for two interesting parameters, unless differently specified.

\section{Results}

Figure 1 shows the LECS+MECS+PDS spectra fitted with a power law model absorbed by the Galactic column density 
Table 2. Spectral fits with a neutral absorber.

\begin{tabular}{lcccccc}
\hline \hline model & $N_{\mathrm{H}}^{a}$ & $z$ & $\alpha_{E}^{b}$ & $\alpha_{\mathrm{H}}^{c}$ & $E_{\text {break }}^{d}$ or $E_{\text {cut }}^{e}$ & $\chi^{2}$ (d.o.f.) \\
\hline PL & $0.16 \pm 0.03$ & 0 FIX & $0.62 \pm 0.03$ & & & $145.9 / 144$ \\
CUTOFFPL & $0.13_{-0.03}^{+0.05}$ & 0 FIX & $0.47_{-0.07}^{+0.11}$ & & $150_{-50}^{+300}$ & $131.8 / 143$ \\
BKNPL & $0.13_{-0.03}^{+0.04}$ & 0 FIX & $0.73_{-0.08}^{+0.32}$ & $1.52 \pm 0.08$ & $5.0_{-1.5}^{+3.0}$ & $136.1 / 142$ \\
Curved & $0.13_{-0.03}^{+0.03}$ & 0 FIX & $0.49_{-0.26}^{+0.09}$ & $3.5_{-1.8}^{+3.0}$ & $36_{-11}^{+19}$ & $131.8 / 142$ \\
\hline
\end{tabular}

${ }^{a}$ In $10^{22} \mathrm{~cm}^{-2}$.

${ }^{b}$ Power law energy index, $F(E) \propto E^{-\alpha_{E}}$.

${ }^{c}$ Hard power law energy index in the BKNPL and Curved models, $F(E) \propto E^{-\alpha_{E}}$ for $E<E_{\text {break }}$ and $F(E) \propto E^{-\alpha_{\mathrm{H}}} \times E_{\text {break }}^{\left(\alpha_{\mathrm{H}}-\alpha_{E}\right)}$ for $E>E_{\mathrm{break}}$. The Curved model is similar to the BKNPL model but the two power laws are joined smoothly.

${ }^{d}$ Break energy in $\mathrm{keV}$ in the BKNPL and Curved models.

${ }^{e}$ Exponential cut-off energy in keV in the CUTOFFPL model, $F(E) \propto E^{-\alpha_{E}} \times \mathrm{e}^{E / E_{\text {cut }}}$.

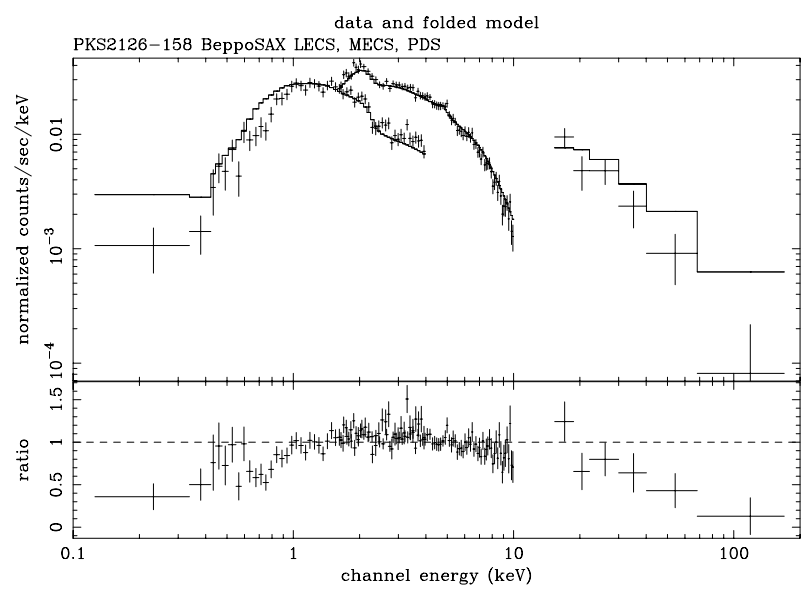

Fig. 1. The LECS, MECS and PDS spectra of PKS 2126-158 fitted with a power law model with Galactic absorption.

along the line of sight $\left(4.85 \times 10^{20} \mathrm{~cm}^{-2}{ }^{1}\right.$ Elvis et al. 1989). The figure shows that the $0.1-100 \mathrm{keV}$ spectrum of PKS 2126-158 is complex. A strong cut-off below $1 \mathrm{keV}$ is clearly visible as well as a broad-band spectral curvature.

To allow an easier comparison with results obtained with other satellites we give in Table 2 the results of a fit with a simple power law model reduced at low energy by the Galactic column density of $4.85 \times 10^{20} \mathrm{~cm}^{-2}$ and by an additional column at zero redshift, assuming solar abundances. The $\chi^{2}$ is acceptable, but relatively large residuals are present at both low $(0.4-0.8 \mathrm{keV})$ and high $(>20 \mathrm{keV})$ energies. Both the spectral index and the absorbing column are consistent with previous ROSAT (Elvis et al. 1994) and ASCA (Serlemitsos et al. 1994; Cappi et al. 1997; Reeves \& Turner 2000) determinations.

Allowing the redshift of the absorber to be free to vary does not improve the fit. Figure 2 shows the $\chi^{2}$ confidence contours for the $N_{\mathrm{H}}$ and the redshift for a power law model continuum. With this choice for the continuum shape we can conclude that absorption in addition to the Galactic value is required by the

\footnotetext{
${ }^{1}$ Cappi et al. (1997) verified that large molecular clouds are not present along the line of sight to PKS 2126-158, and that the Galactic gas column density estimated from the IRAS $100 \mu \mathrm{m}$ maps, assuming a typical gas to dust ratio, is $\lesssim$ the $21 \mathrm{~cm}$ estimate.
}

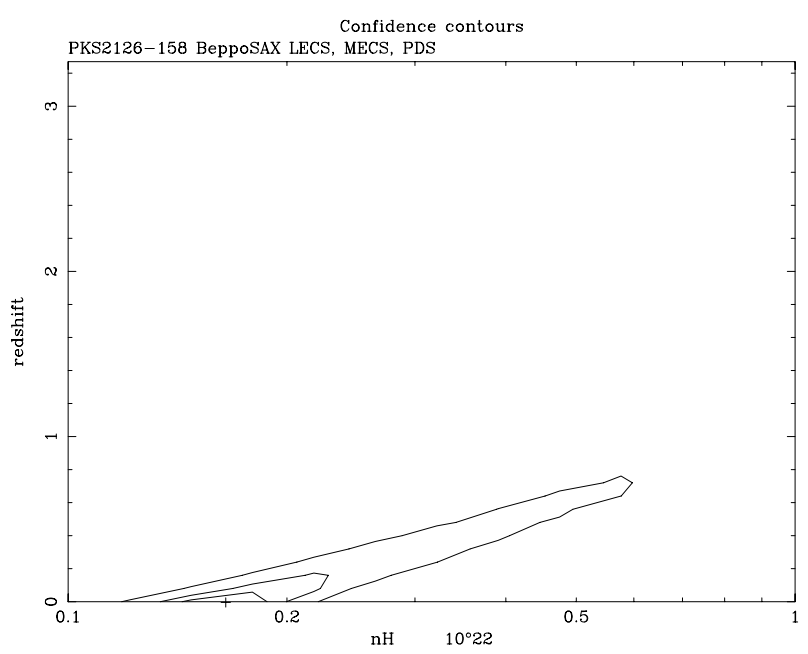

Fig. 2. $N_{\mathrm{H}}$ vs. redshift $\chi^{2} 67 \%, 90 \%$ and $99 \%$ confidence contours for a power law continuum.

data at high confidence level and that the redshift of the absorber must be smaller than 0.8 at the $99 \%$ confidence level.

However, Fig. 1 indicates some curvature of the underlying continuum. This was already suggested by ASCA observations (Serlemitsos et al. 1994; Cappi et al. 1997). Intrinsic curvature of the low energy spectrum will of course result, in low resolution spectra, in a change of the shape of the actual low energy photoelectric cutoff and therefore of the estimated absorber properties (density, redshift, physical and chemical composition).

We have therefore fitted the data using a power law with a high energy exponential cutoff (see Table 2). The improvement in $\chi^{2}$ with respect to the simple power law model is significant at $>99.99 \%$ confidence level. The ratio between the data and the best fit model is shown in Fig. 3. Allowing the redshift of the absorber be free to vary does not improve the fit. Figure 4 shows the $N_{\mathrm{H}}$ vs. redshift $\chi^{2}$ confidence contours for the power law with exponential cutoff model continuum.

We have also fitted the data using a broken power law model and with with a broken power law model with a smooth conjunction between the two power laws ("Curved" model). The results are again in Table 2. For the broken power law model The improvement in $\chi^{2}$ with respect to the simple 


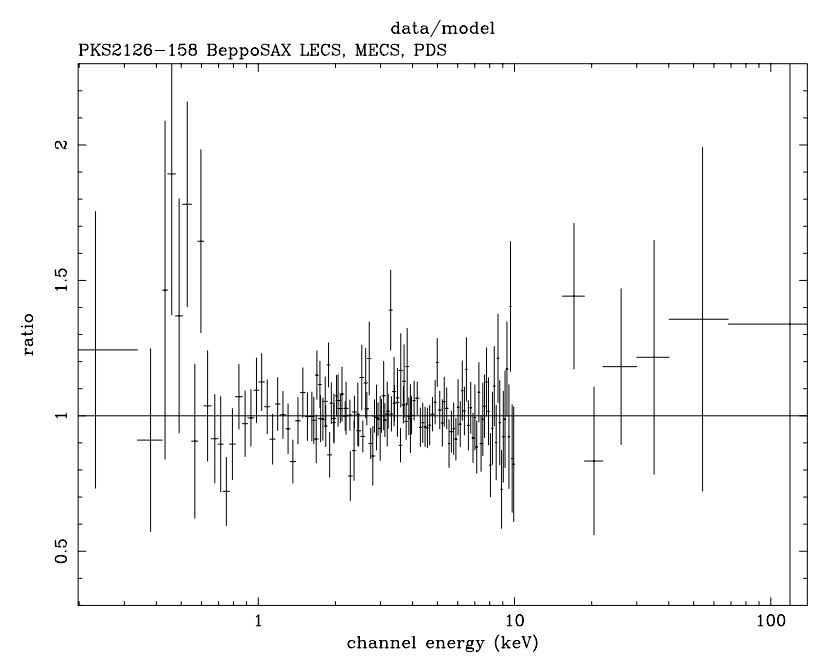

Fig. 3. Ratio between the BeppoSAX LECS, MECS and PDS spectra of PKS 2126-158 and the best fitting power law with exponential cutoff model.

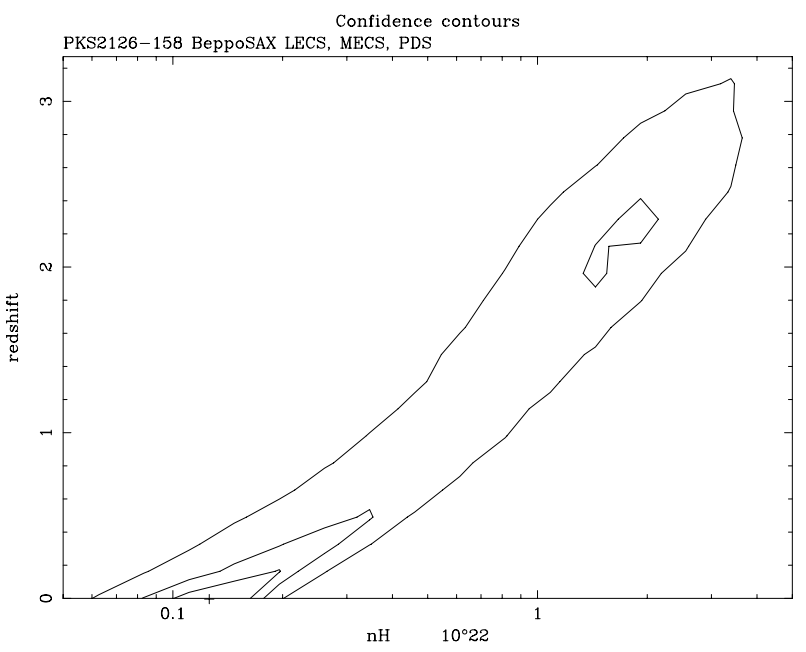

Fig. 4. $N_{\mathrm{H}}$ vs. redshift $\chi^{2} 67 \%, 90 \%$ and $99 \%$ confidence contours for a broken power law continuum.

power law model is significant at the $99.28 \%$ confidence level. Figure 5 shows the $N_{\mathrm{H}}$ vs. redshift $\chi^{2}$ confidence contours for a broken power law model continuum. There are two minima in the $\chi^{2}$ surface, one at $z=0$ and the second at $z \sim 2.2$. The best fit results for the "curved" model resemble those of the power law with exponential cutoff model, but with an additional free parameter.

Figures 4 and 5 show that if the absorption is neutral and the continuum is parameterized with either a broken power law or a power law with a cutoff, the redshift of the absorber is less that that of the quasar at better than the $99 \%$ confidence level.

For the three continuum models, we have also fitted an ionized absorber model (ABSORI in XSPEC) at both $z=0$ and $z=3.27$. The results are given in Table 3. The continuum parameters are very close to those in Table 2 and therefore, for simplicity, we give in Table 3 the parameters of the absorber only. The fits with the absorber at $z=0$ give a good $\chi^{2}$ in all cases. Equally good fits for an absorber at the quasar redshift can only be obtained if the iron abundance $Z_{\mathrm{Fe}}$ is

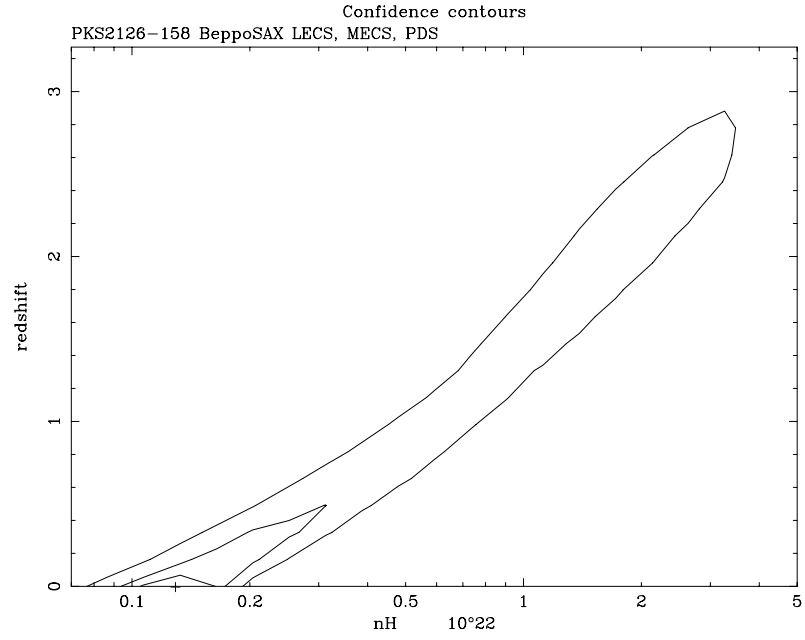

Fig. 5. $N_{\mathrm{H}}$ vs. redshift $\chi^{2} 67 \%, 90 \%$ and $99 \%$ confidence contours for a power law with exponential cutoff continuum.

substantially less than solar. (The iron $\mathrm{K}$ edges are redshifted into the $1.6-2.0 \mathrm{keV}$ band. At these energies the quality of the LECS and MECS spectra can strongly constrain the iron abundance.) Since the abundance of all other elements is fixed to the solar value, the fits actually constrain the $[\alpha / \mathrm{Fe}]$ ratio to be higher than 0.5 .

As an example Fig. 6 shows the $\chi^{2}$ confidence contours for the iron abundance versus the ionization parameter, for a power law continuum model, assuming that the absorber is at the redshift of the quasar. The analogous contours for the other two parameterizations of the continuum are similar. In all cases the best fit solution implies column densities of the order of a few $\times 10^{23} \mathrm{~cm}^{-2}$, high ionization parameter and low iron abundance. The inclusion of an additional cold absorber at the quasar redshift does not improve the $\chi^{2}$.

\section{Discussion}

\subsection{Flux, luminosity and variability}

The $2-10 \mathrm{keV}$ and $0.1-2.5 \mathrm{keV}$ fluxes observed by BeppoSAX are $1.1 \times 10^{-11}$ and $4.4 \times 10^{-12} \mathrm{erg} \mathrm{cm}^{-2} \mathrm{~s}^{-1}$ respectively. The unabsorbed $0.1-2.5 \mathrm{keV}$ flux is 2.1 times higher $(1.0 \times$ $\left.10^{-11} \mathrm{erg} \mathrm{cm}^{-2} \mathrm{~s}^{-1}\right)$. The statistical uncertainties on these values is of the order of a few \%, smaller than the systematic uncertainty in the LECS and MECS flux absolute calibration (which is of the order of 5\%, Sacco, private communication, also see Fiore et al. 1999). The unabsorbed fluxes correspond to a $2-10 \mathrm{keV}$ and $0.1-2.5 \mathrm{keV}$ luminosities of $1.6 \times 10^{48} \mathrm{erg} \mathrm{s}^{-1}$ and $1.5 \times 10^{48} \mathrm{erg} \mathrm{s}^{-1}$ respectively, assuming "isotropic" emission and using $H_{0}=50$ and $\Omega_{0}=0$. For a $H_{0}=65, \Omega_{0}=0.3$ and $\Omega_{\Lambda}=0.7$ cosmology the $2-10 \mathrm{keV}$ and $0.1-2.5 \mathrm{keV}$ luminosities are 2.5 times smaller $\left(6.8 \times 10^{47} \mathrm{erg} \mathrm{s}^{-1}\right.$ and $6.2 \times$ $10^{47} \mathrm{erg} \mathrm{s}^{-1}$ respectively).

The observed fluxes are compared in Fig. 7 with previous measurements obtained with the Einstein, EXOSAT, ROSAT and ASCA satellites (Worrall \& Wilkes 1990; Elvis et al. 1994; Cappi et al. 1997) and with the fluxes recorded in two 
Table 3. Spectral fits with an ionized absorber ${ }^{a}$.

\begin{tabular}{lccccc}
\hline \hline model & $N_{\mathrm{H}}^{b}$ & $z$ & $\xi^{c}$ & $Z_{\mathrm{Fe}}^{d}$ & $\chi^{2}$ (d.o.f.) \\
\hline PL & $0.26 \pm 0.05$ & 0 FIX & $0.21_{-0.20}^{+0.50}$ & $1 \mathrm{FIX}$ & $134.7 / 143$ \\
BKNPL & $0.23_{-0.10}^{+0.20}$ & 0 FIX & $<0.8$ & 1 FIX & $131.4 / 140$ \\
CUTOFFPL & $0.20_{-0.06}^{+0.10}$ & 0 FIX & $<1.0$ & 1 FIX & $126.5 / 142$ \\
\hline PL & $50_{-25}^{+150}$ & 3.27 FIX & $1800_{-1400}^{+2500}$ & $<0.25$ & $140.8 / 142$ \\
BKNPL & $36_{-24}^{+150}$ & 3.27 FIX & $1300_{-1100}^{+3700}$ & $<0.25$ & $129.5 / 141$ \\
CUTOFFPL & $36_{-24}^{+50}$ & 3.27 FIX & $1700_{-1500}^{+3800}$ & $<0.3$ & $127.6 / 141$ \\
\hline
\end{tabular}

${ }^{a}$ Continuum fit values are indistinguishable from those in Table 2.

${ }^{b}$ In $10^{22} \mathrm{~cm}^{-2} ;{ }^{c}$ ionization parameter. ${ }^{d}$ Iron abundance in unit of the solar value.

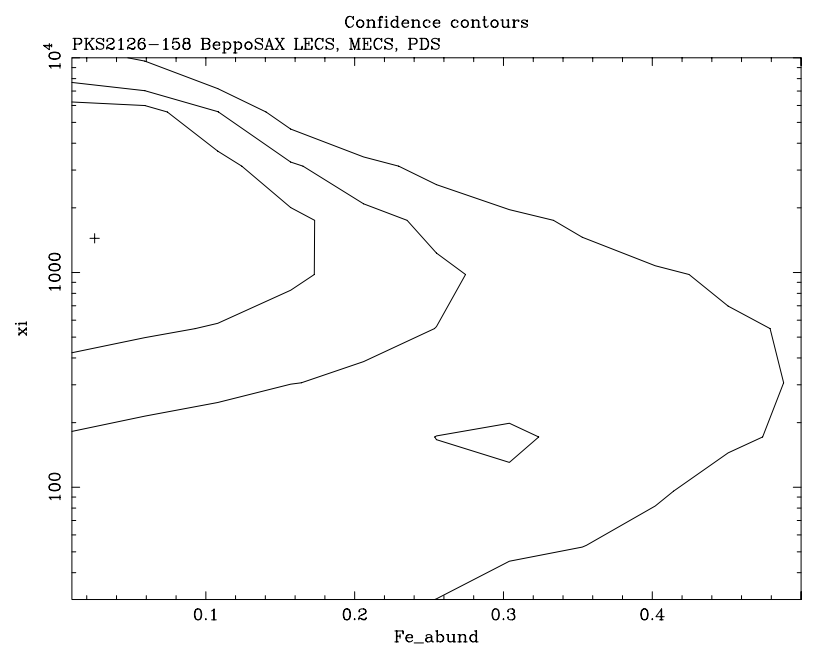

Fig. 6. Ionization parameter vs. iron abundance $\chi^{2} 67 \%, 90 \%$ and $99 \%$ confidence contours for a power law with exponential cut-off continuum.

recent Chandra and XMM-Newton observations (D'elia et al. 2003 in preparation, Ferrero \& Brinkmann 2003). 5\% systematic errors have been added in quadrature to the statistical errors on the fluxes. Figure 7 shows that BeppoSAX caught the source in its historical maximum, more than a factor of 2 brighter than all previous observation and $40 \%$ brighter than in the later Chandra and XMM-Newton observations. A detailed analysis of the source spectral variability among the ASCA, BeppoSAX, Chandra and XMM-Newton observation is beyond the scope of this paper and will be presented elsewhere (D'Elia et al. 2003). The rest frame time delay between the ASCA and BeppoSAX observations is about one year, while that between the BeppoSAX and Chandra and BeppoSAX and XMM-Newton observations is of 1.5 and 6 months respectively.

PKS 2126-158 is a GigaHertz Peaked Spectrum (GPS) quasar (O'Dea et al. 1991; Stanghellini et al. 2001). On a 3 miliarcsec scale the source has core-jet morphology, with a possible counter jet detection. Based on the possible counter jet detection and, more importantly, on the low polarization, Stanghellini et al. (2001) conclude that PKS 2126-158 can be only marginally beamed. If beaming is not large in this source, then the above "isotropic" luminosities are likely to

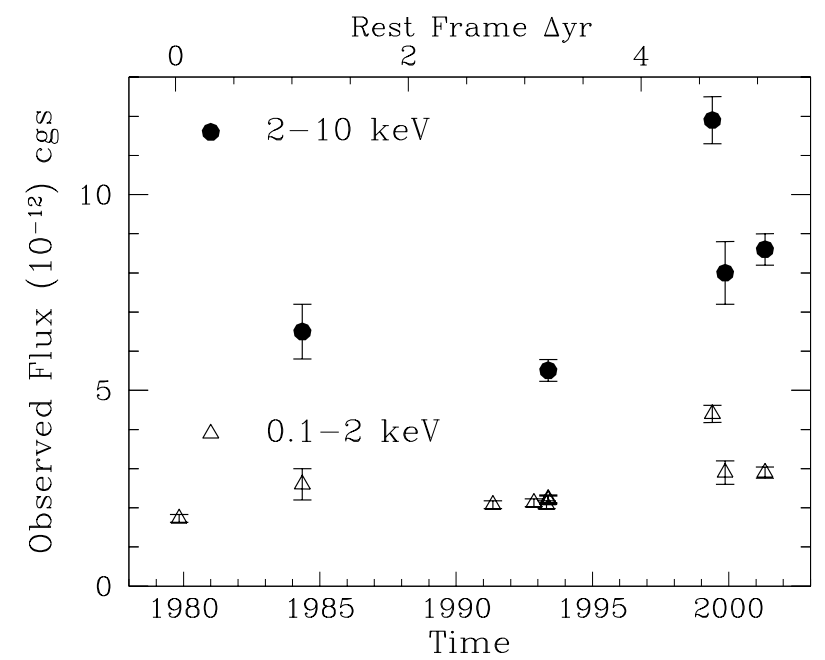

Fig. 7. $2-10 \mathrm{keV}$ (filled circles) and $0.1-2 \mathrm{keV}$ (open triangles) fluxes observed by Einstein, EXOSAT, ROSAT, ASCA, BeppoSAX and XMM-Newton in the last $22 \mathrm{yrs}$. Error bars are $1 \sigma$ statistical uncertainties.

be close (within a factor of a few) to the real luminosities. These then would imply extreme black hole masses of at least a few $\times 10^{10} M_{\odot}$, assuming that the quasar is emitting close to its Eddington luminosity. The causal timescale associated to an emission region of $\sim 10$ Schwarzschild radii around these black holes is $\sim$ months, comparable to the shortest variability timescale detected.

The absorbing column density depends of course by the redshift. If $z=0$ is assumed then the BeppoSAX values in Table 2 are well consistent with the ROSAT and ASCA ones. If $z=3.27$ is assumed then the column densites in Figs. 4 and 5 $\left(2-4 \times 10^{22} \mathrm{~cm}^{-2}\right)$, are somewhat higher than the ROSAT and ASCA values (Elvis et al. 1994; Cappi et al. 1997), but still statistically compatible with them. We note that this slight discrepacy is likely due to the fact that the continuum assumed by the latter authors is a simple power law, while Figs. 4 and 5 are obtained using a broken power law and a power law with an exponential cutoff continuum. 


\subsection{Low energy absorption}

The BeppoSAX NFI observations of PKS 2126-158 confirm the presence of low energy absorption in this source independent of the continuum form. Unlike all previous investigations, even assuming a curved continuum and a broken power law continuum, the spectra do require, at a high confidence level, absorption in excess to the Galactic column density along the line of sight. Because of the limited spectral resolution of the LECS and MECS it is difficult to distinguish between different absorption and continuum models and to assess precisely the redshift of the absorber. If a power law model is used to parameterize continuum the redshift of the absorber is smaller than 0.8 at the $99 \%$ confidence level. If the continuum is parameterized using a broken power law the redshift of the absorber is more poorly constrained (the $99 \% \chi^{2}$ contours nearly touch the emission redshift of 3.27). Interestingly there are two minima in the $\chi^{2}$ surface, one at $z=0$ and the second at $z=2.2$. If the continuum is parameterized using a power law with an exponential cutoff then $z_{\text {abs }}<3$ at the $99 \%$ confidence level. In summary, if the absorber is not strongly ionized, the BeppoSAX data do prefer an absorber at $z<z_{\mathrm{em}}$. X-ray absorption up to a level of $10^{22} \mathrm{~cm}^{-2}$ can be produced by DLA systems along the line of sight. This is seen in several low- $z$ DLAs by Chandra (Bechtold et al. 2001; Siemiginowska et al. 2002). Although, no DLA is observed toward PKS 2126-158, only a small part of the redshift range is accessible to direct DLA searches, since a $z=2.9676$ Lyman limit system cuts out shorter wavelength UV emission, so DLAs cannot be directly detected below $z=1.976$. However, several complex metal line systems have been detected at $0.66<z<2.9$ (Giallongo et al. 1993; D'odorico et al. 1998). In particular, the system at $z=0.6631$ has strong low ionization CaII3934-3969 and MgII2796-2803 absorption. The MgII column is $4( \pm 1) \times 10^{17} \mathrm{~cm}^{-2}$ (D'odorico et al. 1998), which would correspond to a hydrogen column of $\sim 10^{22} \mathrm{~cm}^{-2}$ for solar abundances, assuming that the MgII ion is the most populated of the $\mathrm{Mg}$ ions. Since other $\mathrm{Mg}$ ions will likely to be populated, the hydrogen column could actually be higher than this value. Therefore a DLA could well be associated to the 0.6631 system and could be responsible for the X-ray absorption. Strong, $\tau>1$, low ionization oxygen edges at $\sim 0.33 \mathrm{keV}$ are predicted if $N_{\mathrm{H}} \gtrsim 10^{22} \mathrm{~cm}^{-2}$ and if the gas ionization is as low, as suggested by the strong MgII line. Furthermore, at $z=2.63-2.82$ there are 5 metal systems within a velocity range of $800 \mathrm{~km} \mathrm{~s}^{-1}$ (Giallongo et al. 1993). They clearly indicate a rich environment at this redshift in the direction of PKS 2126-158, which could again well be associated with the site of the X-ray absorption.

On the other hand, a highly ionized absorber at the quasar redshift provides a good fit only if the iron abundance is smaller than $\sim 0.3$ solar, while those of all other elements are fixed to the solar value. The ionized absorber could be the result of the interaction of the radio jet of PKS 2126-158 with the ISM of the host galaxy. PKS 2126-158 is a GPS quasar and GPS sources are often explained in term of a young, newly born radio sources. Silk \& Rees (1998) and Fabian (1999) proposed a scenario in which newly born, high luminosity quasars, are still enshrouded by large densities of soft $\mathrm{X}$-ray absorbing gas.
If its X-ray absorption is intrinsic PKS 2126-158, could then be an example of these newly born, highly obscured quasars. The connection of X-ray cut-offs and newly born quasars is highly suggestive, and it must be studied in detail with future high resolution X-ray observation, which can be able to constrain tightly the redshift of the absorbers.

In the ionized absorber solution the limit to the iron abundance is rather tight $\left(Z_{\mathrm{Fe}}<0.3\right)$. This is due to the good sensitivity of the LECS and MECS at $1.5-2 \mathrm{keV}$, where the $\mathrm{Fe}-\mathrm{K}$ edges are redshifted. This limit contrasts with the estimate of the nuclear iron abundance obtained by Thompson et al. (1999) using the intensity of iron optical emission lines. However, as mentioned in the introduction optical estimates may be inaccurate. The X-ray metallicity determination and optical lines measurements could be combined to constrain the line emitting gas physical status. An underabundance of iron $([\alpha / \mathrm{Fe}]>0.5)$, would be consistent with a scenario in which the ISM has been enriched mostly by type II SNe. If confirmed, this could be used to put constraints on the time needed to form the bulk of type Ia SNe, i.e. the time to form a WD in a binary system plus the time needed to accrete matter from the companion to surpass the Chandrasekar limit, which brings to the $\mathrm{SN}$ explosion. All these time-scales are today rather poorly defined.

Since iron aggregates easily in dust grains, an iron abundance of the X-ray absorbing matter significantly less than the solar value can contribute to explaining the lack of strong ultraviolet reddening in this source (Elvis et al. 1994; Bechtold et al. 1994), expected from the measured X-ray absorbing column density, if the dust to gas ratio and dust composition are similar to the Galactic one. A low iron abundance absorber can also help in solving the apparent paradox of hard X-ray selected high redshift quasars which appear to be obscured in X-rays but show strong broad optical and UV lines and little extinction in the optical band (see Fiore et al. 2001; Comastri et al. 2001; Maiolino et al. 2001a). Other possible solutions of these contradictions are: (1) the X-ray absorber could be highly ionized and the dust destroyed, if the matter is close enough to the active nucleus; (2) the dust grains could have different properties with respect to the "standard" Galactic mixture (Maiolino et al. 2001b).

Although the ionized absorber hypothesis is statistically tenable and astrophysically intriguing, in that it helps in resolving the contradiction between the X-ray absorption and the lack of UV extinction, the present low resolution data are inadequate to choose this as a unique solution. The precise determination of the absorber redshift, as well as of its metallicity and ionization state must await for the high resolution observations that have become possible with the gratings on board of the Chandra and XMM-Newton satellites.

Acknowledgements. We would like to thank the BeppoSAX hardware teams for the development and calibration of the instruments. In particular, we would like to remember the late Daniele Dal Fiume for his continuous dedication and his fundamental contribution to an instrument of unprecedented sensitivity like the BeppoSAX PDS. We thank E. Giallongo for useful discussions. This research has made use of SAXDAS linearized and cleaned event files (Rev.2.0) produced at the 
BeppoSAX Science Data Center. This research has been partially supported by ASI contract ARS-99-75 and MURST grant Cofin-98-032.

This research has made use of the NASA/IPAC Extragalactic Database (NED) which is operated by the Jet Propulsion Laboratory, Caltech, under contract with the National Aeronautics and Space Administration, and the HEASARC Databases.

\section{References}

Arav, N., de Kool, M., Korista, K. T., et al. 2001, ApJ, 561, 118

Bechtold, J., Elvis, M., Fiore, F., et al. 1994, AJ, 108, 374

Bechtold, J., Siemiginowska, A., Aldcroft, T. L., Elvis, M., \& Dobrzycki, A. 2001, ApJ, 562, 133

Boella, G., Butler, R. C., Perola, G. C., et al. 1997a, A\&AS, 122, 299

Boella, G., Chiappetti, L., Conti, G., et al. 1997b, A\&AS, 122, 327

Cappi, M., Matsuoka, M., Comastri, A., et al. 1997, ApJ, 478, 492

Churchill, C. W., Mellon, R. R., Charlton, J. C., et al. 2000, ApJS, 130,91

Constantin, A., Shields, J. C., Hamann, F., Folz, C. B., \& Chaffee, F. H. 2002, ApJ, 565, 50

Dietrich, M., Appenzeller, I., Vestergaard, M., \& Wagner, S. J. 2002, ApJ, 564, 581

D’Odorico, V., Cristiani, S., D’Odorico, S., Fontana, A., \& Giallongo, E. 1998, A\&AS, 127, 217

Elvis, M., Lockman, F. J., \& Wilkes, B. J. 1989, AJ, 97, 777

Elvis, M., Fiore, F., Wilkes, B. J., McDowell, J. C., \& Bechtold, J. 1994, ApJ, 422, 60

Elvis, M., Fiore, F., Giommi, P., \& Padovani, P. 1998, ApJ, 492, 91

Fabian, A. C. 1999, MNRAS, 308, L39

Ferrero, E., \& Brinkmann, W. 2003, A\&A, 402, 465

Fiore, F., Elvis, M., Giommi, P., \& Padovani, P. 1998, ApJ, 492, 79

Fiore, F., Guainazzi, M., \& Grandi, P. 1999, Handbook for BeppoSAX NFI spectral analysis,

ftp://ftp.asdc.asi.it/pub/sax/doc/software_docs/ saxabc_v1.2.ps.gz/ or http://heasarc.gsfc.nasa.gov/docs/sax/abc/saxabc/

Frontera, F., Costa, E., dal Fiume, D., et al. 1997, A\&AS, 112, 357

Giallongo, E., Cristiani, S., Fontana, A., \& Trevese, D. 1993, ApJ, 416,137
Hamann, F. 1998, ApJ, 500, 798

Hamann, F., \& Ferland, G. J. 1999, ARA\&A, 37, 487

Hamann, F., Korista, K. T., Ferland, G. J., Warner, C., \& Baldwin, J. 2002, ApJ, 564, 592

Hamann, F., Sabra, B., Junkkarinen, V., Cohen, R., \& Shields, G. 2003, X-ray spectroscopy of AGN with Chandra and XMM, Proc. of the workshop Garching, Germany, December 2001 [astro-ph/0304564]

Kuhn, O., Elvis, M., Bechtold, J., \& Elston, R. 2001, ApJS, 136, 225

Iwamuro, F., Motohara, K., Maihara, T., et al. 2002, ApJ, 565, 63

Levine, A. M., Lang, F. L., Lewin, W. H. G., et al. 1984, ApJS, 54, 581

Madejski, G., Takahashi, T., Tashiro, T., et al. 1996, ApJ, 459, 156

Maiolino, R., Marconi, A., Salvati, M., et al. 2001a, A\&A, 365, 28

Maiolino, R., Marconi, A., \& Oliva, E. 2001b, A\&A, 365, 37

Nicastro, F., Fiore, F., Perola, G. C., \& Elvis, M. 1999a, ApJ, 512, 184

Nicastro, F., Fiore, F., Perola, G. C., \& Elvis, M. 1999b, ApJ, 512, 136

O’Dea, C. P., Baum, S. A., \& Stanghellini, C. 1991, ApJ, 380, 66

Parmar, A., Martin, D. D. E., Bavdaz, M., et al. 1997, A\&AS, 112, 309

Pettini, M., Smith, L. J., King, D. L., \& Hunstead, R. W. 1997, ApJ, 486, 665

Pettini, M., Ellison, S. L., Steidel, C. C., \& Bowen, D. V. 1999, ApJ, 510,576

Prochaska, J. X., \& Wolfe, A. M. 1999, ApJS, 121, 369

Reeves, J. N., \& Turner, M. J. L. 2000, MNRAS, 316, 234

Savaglio, S., Panagia, N., \& Stiavelli, M. 2000, ed. J. Franco, L. Terlevich, O. Lopez-Cruz, \& I. Aretxaga, Astronomical Society of the Pacific, ASP Conf. Proc., 215, 65 [astro-ph/0011473]

Serlemitsos, P. Yaqoob, T., Ricker, G., et al. 1994, PASJ, 46, L43

Silk, J., \& Rees, M. 1998, A\&A, 331, L1

Stanghellini, C., Dallacasa, D., O’Dea, C. P., et al. 2001, A\&A, 377, 377

Thompson, K. L., Hill, G. J., \& Elston, R. 1999, 515, 487

Verner, E. M., Verner, D. A., Korista, K. T., et al. 1999, ApJS, 120, 101

Worral, D. M., \& Wilkes, B. J. 1990, ApJ, 360, 396 